\title{
Nutritional rehabilitation of mitochondrial aberrations in aplastic anaemia
}

\author{
Ling $\mathrm{He}^{1}$, Xiaoyan Miao ${ }^{1}$, Guangyan $\mathrm{Lv}^{2}$, Peiman Yang ${ }^{3}$, Wenguo $\mathrm{Wu}^{4}$ and $\mathrm{Li} \mathrm{Jia}^{1 *}$ \\ ${ }^{1}$ College of Laboratory Medicine, Dalian Medical University, 9 Lushunnan Road Xiduan, Dalian 116044, Liaoning \\ Province, China \\ ${ }^{2}$ Central Laboratory, Dalian Medical University, Dalian 116044, Liaoning Province, China \\ ${ }^{3}$ Department of Histology and Embryology, Dalian Medical University, Dalian 116044, Liaoning Province, China \\ ${ }^{4}$ ZHEN-AO Group Company, Dalian, Liaoning Province, China \\ (Received 17 May 2010 - Revised 18 October 2010 - Accepted 19 October 2010 - First published online 14 December 2010)
}

\begin{abstract}
Aplastic anaemia (AA) is a disease characterised by bone marrow hypocellularity and peripheral blood pancytopenia. AA is also associated with mitochondrial aberrations. The present study was undertaken primarily to test the hypothesis that a nutrient mixture could affect the nutritional rehabilitation of mitochondrial aberrations in AA mice. BALB/c AA mice were induced by a combination of hypodermic injections of acetylphenylhydrazine $(100 \mathrm{mg} / \mathrm{kg})$, X-rays $(2 \cdot 0 \mathrm{~Gy})$ and intraperitoneal injections of cyclophosphamide $(80 \mathrm{mg} / \mathrm{kg})$. We treated these mice with nutrient mixture-supplemented diets in a dose-dependent manner $(1445 \cdot 55,963 \cdot 7,674 \cdot 59 \mathrm{mg} / \mathrm{kg}$ per d), and the effects of the nutrient mixture for mitochondrial rehabilitation were analysed in AA mice. Transmission electron microscopy showed that mitochondrial ultrastructural abnormalities in bone marrow cells, splenocytes and hepatocytes of the nutrient mixture groups were restored markedly, compared with the AA group. Mitochondrial membrane potentials of the nutrient mixture groups were increased remarkably. Western blot analysis also revealed that the nutrient mixture significantly inhibited cytochrome $c$ release of mitochondria in the AA group. Furthermore, the mitochondrial DNA content of the nutrient mixture groups was also increased. Our data suggest that the nutrient mixture may promote the rehabilitation of mitochondrial aberrations, and consequently protects against mitochondrial dysfunction in AA mice.
\end{abstract}

Key words: Nutrient mixture: Aplastic anaemia: Mitochondria

Aplastic anaemia (AA) is a serious medical disorder that is characterised by bone marrow hypocellularity and peripheral blood pancytopenia. The major signs and symptoms are exhaustion, infections and bleedings ${ }^{(1)}$. AA is a heterogeneous disease, which involves destruction of haematopoietic stem cells, disturbance of haematopoietic microenvironment, immune-mediated reaction against haematopoietic tissue or a combination of factors ${ }^{(2)}$. Clinical and experimental evidence suggests that AA has been strongly associated with exposure to environmental factors including radiation, drugs and chemicals and viruses ${ }^{(1)}$. In our experiments, the AA mouse model was induced by a combination of acetylphenylhydrazine, X-rays and cyclophosphamide, and this model shared many similarities with the human condition of $\mathrm{AA}^{(3-5)}$.

Mitochondria are double-membrane organelles that are present in the cytoplasm of most eukaryotic cells. Mitochondria generate most of the cellular ATP via oxidative phosphorylation in an oxygen-dependent process. In addition to supplying cellular energy, mitochondria are involved in cell signalling, cellular differentiation and apoptosis, as well as in the control of the cell cycle and cell growth. The number of mitochondria in a cell varies widely by organism and tissue type ${ }^{(6)}$. Mitochondrial dysfunction is a hallmark of almost all diseases, including cancer and multiple neurodegenerative diseases ${ }^{(7)}$. Mitochondria are under the dual genetic control of both mitochondrial genome (mtDNA) and nuclear $\mathrm{DNA}^{(8)}$. Mutations in mtDNA may be associated with some haematological diseases, including acquired sideroblastic anaemia, myelodysplastic syndromes and acquired $\mathrm{AA}^{(9-11)}$.

Nutritional rehabilitation has emerged as a promising component of comprehensive treatment and rehabilitation. It plays a significant role in improving metabolism, enhancing immunity of patients and maintaining normal physiology. Our previous data showed that a nutrient mixture played a key role in the nutritional rehabilitation of AA mice. Here, we first employed the AA mouse model to investigate the role by which a nutrient mixture mediates the rehabilitation of mitochondrial aberrations.

Abbreviations: AA, aplastic anaemia; GSE, grape seed extract; MMP, mitochondrial membrane potential; MtDNA, mitochondrial DNA; RPMI-1640, Roswell Park Memorial Institute 1640; Rh123, rhodamine 123.

*Corresponding author: Dr Li Jia, email jiali0386@hotmail.com 
A nutrient mixture (containing nucleotides, arginine, phosphatidylcholine, phosphatidylethanolamine, $\mathrm{Mn}, \mathrm{Zn}$, vitamin, folate, grape seed extract (GSE) powder, etc.) was administered to AA mice in a dose-dependent manner by intragastric administration over a $45 \mathrm{~d}$ dosing period. This experiment was devised to use the nutrientmixture-treated AA mouse model to examine the changes in mitochondrial aberrations. The present results show that the nutrient mixture can promote the rehabilitation of mitochondrial aberrations in AA mice.

\section{Materials and methods \\ Animals and treatments}

Male BALB/c mice (8 weeks old) were obtained from Animal Facility of Dalian Medical University. Animals were individually housed in plastic cages and placed in an air-conditioned room with a $12 \mathrm{~h}$ light- $12 \mathrm{~h}$ dark cycle, at a temperature of $20 \pm 2^{\circ} \mathrm{C}$ and a relative humidity of $55 \pm 10 \%$. They were maintained under specific pathogen-free conditions in the Animal Facility of Dalian Medical University and were fed ad libitum with the standard diet and water throughout the experimental period.

After a 1-week adaptation period, 100 mice were randomly divided into five groups with twenty in each group: the control group; the AA group; the three different doses of the nutrient-mixture-supplemented diet groups. The mice of the AA model group and of the three different doses of the nutrient mixture groups were treated with a combination of hypodermic injections of acetylphenylhydrazine $(100 \mathrm{mg} / \mathrm{kg}$; Sigma, St Louis, MO, USA), X-rays $(2.0 \mathrm{~Gy})$ and intraperitoneal injections of cyclophosphamide $(80 \mathrm{mg} / \mathrm{kg}$; Sigma). The control group was treated with lead shielding irradiation alone and injections of physiological saline. After the 7th day, the mice of the nutrient mixture groups were given a nutrient-mixturesupplemented diet in a dose-dependent manner (1445.55, $963 \cdot 7,674.59 \mathrm{mg} / \mathrm{kg}$ per $\mathrm{d}$, according to a Chinese Medical Dictionary) by intragastric administration. The control and AA model groups were given a physiological saline ( $10 \mathrm{ml} / \mathrm{kg}$ per d)-supplemented diet by intragastric administration. During the supplemented dosing period, body weights of animals were determined daily throughout the course of the experiment.

After 7 weeks, mice were killed by cervical dislocation, and then the following physiological conditions were determined: blood, mitochondria of bone marrow, spleen and liver cells, mitochondrial membrane potential (MMP), mtDNA content and release of cytochrome $c$. All animal experiments were performed according to the guidelines for the care and use of animals approved by the Dalian Medical University.

The nutrient mixture was supplied by Zhen-Ao Group Co., Ltd (Dalian, China) and composed of nucleotides $(22 \cdot 8 \%)$, arginine $(5 \cdot 7 \%)$, lysine $(5.7 \%)$, cysteine $(3.4 \%)$, glycine $(6 \cdot 8 \%)$, histidine $(5 \cdot 7 \%)$, lecithin $(27 \cdot 4 \%)$, cephalin (13.6\%), vitamin mix (containing vitamin $\mathrm{E}$, vitamin $\mathrm{C}$, vitamin $\mathrm{B}_{6}$, vitamin $\mathrm{B}_{12}$ and folate) (1.58\%), $\mathrm{Zn}(0 \cdot 13 \%), \mathrm{Mn}$ $(0 \cdot 11 \%)$, Fe $(0 \cdot 28 \%)$, medlar polysaccharides $(3 \cdot 4 \%)$ and GSE $(3 \cdot 4 \%)$. According to the proto-prescription, oral application was prepared by decocting, ultrasound and concentrating.

\section{Analysis of peripheral blood}

Blood was obtained from mice and mixed immediately with dipotassium EDTA (Sigma). Blood samples were analysed using the Sysmex KX-21N Blood Cell analyser (Kobe, Japan).

\section{Transmission electron microscopy studies}

Fresh tissues (bone marrow, spleen and liver) were processed for transmission electron microscopy. These samples were first washed with phosphate-buffered saline for $5 \mathrm{~min}$, fixed with $2 \cdot 0 \%$ glutaraldehyde in $0.1 \mathrm{M}$ phosphate buffer ( $\mathrm{pH} 7 \cdot 4)$, washed in $0 \cdot 1 \mathrm{M}$ phosphate buffer and post-fixed in $1.0 \%$ osmium tetroxide in $0.1 \mathrm{~m}$ phosphate buffer ( $\mathrm{pH} 7 \cdot 4)$. The samples were then dehydrated and were finally embedded in Epon 812 solution for ultrathin sectioning. The ultrathin sections were stained with uranyl acetate and lead citrate and were viewed with a JEM-2000EX transmission electron microscope at a voltage of $120 \mathrm{kV}$. Transmission electron microscopic examinations of ultrathin sections were under 100 fields of vision.

\section{Cell isolation and culture}

Under aseptic conditions, femora were removed, with the surrounding muscle, and placed in $5 \mathrm{ml}$ of sterile $90 \%$ Roswell Park Memorial Institute 1640 (RPMI-1640) medium (Gibco, Grand Island, NY, USA), supplemented with $10 \%$ fetal bovine serum (Gibco). Then, the muscle and epiphyses were removed from femora and marrow and flushed into $5 \mathrm{ml}$ of sterile 90\% RPMI-1640 medium supplemented with $10 \%$ fetal bovine serum.

Spleens and livers were dissected from mice, followed by the preparation of single cell suspensions under aseptic conditions. They were dissected and cut into small pieces, rinsed twice with phosphate-buffered saline and minced with forceps and scalpel. The suspension was passed through a $100 \mu \mathrm{m}$ stainless-steel mesh, treated with a hypotonic buffer $\left(0 \cdot 15 \mathrm{M}-\mathrm{NH}_{4} \mathrm{Cl}, 0.01 \mathrm{M}-\mathrm{KHCO}_{3}, 0 \cdot 1 \mathrm{~mm}-\mathrm{Na}_{2} \mathrm{EDTA}\right.$, $\mathrm{pH} 7 \cdot 4$ ) for $5 \mathrm{~min}$ at room temperature to lyse erythrocytes, centrifuged (1200 rpm, $10 \mathrm{~min}$ ) and washed twice with cell medium (90\% RPMI-1640 supplemented with 10\% fetal bovine serum and $1 \%$ antibiotic). Splenocytes were incubated in dishes for $2 \mathrm{~h}$ to remove adherent cells, and the cells obtained were cultured in cell medium. Hepatocytes were incubated in cell medium (90\% RPMI-1640 
containing $0.05 \%$ collagenase, supplemented with $10 \%$ fetal bovine serum and $1 \%$ antibiotic).

The viability of the cells was determined by trypan blue (Sigma) exclusion, and cell density was adjusted to $5 \times 10^{5}$ cells/ml for the MMP assay.

\section{Mitochondrial membrane potential assay}

Rhodamine 123 (Rh123) is a voltage-sensitive mitochondrial dye that is able to enter the mitochondrial matrix and accumulate in the mitochondria according to the MMP. The mitochondrial uptake of Rh123 has been measured to determine the $\mathrm{MMP}^{(12)}$. Bone marrow cells from the mice were harvested and washed thrice with phosphate-buffered saline. The cells were resuspended to $5 \times 10^{5}$ cells $/ \mathrm{ml}$ in fresh incubation medium containing $1.0 \mu \mathrm{M}-\mathrm{Rh} 123$ and incubated at $37^{\circ} \mathrm{C}$ in a thermostatic bath for $10 \mathrm{~min}$ with gentle shaking. They were then separated by centrifugation, and the amount of Rh123 remaining in the incubation medium was measured using a fluorescence spectrophotometer set (Hitachi 650-60; Tokyo, Japan) at $490 \mathrm{~nm}$ excitation and $520 \mathrm{~nm}$ emission wavelengths. Results are shown as the fluorescence retained within the cells.

Changes in the MMP of splenocytes and hepatocytes were examined according to the methods described earlier.

\section{Isolation of mitochondria and cytosol}

Mitochondria were extracted from bone marrow-nucleated cells, splenocytes and hepatocytes according to the manufacturer's protocol of the KeyGen mitochondria isolation kit. The cells were harvested and washed twice with phosphate-buffered saline for $5 \mathrm{~min}$ at $800 \mathrm{~g}$ at $4^{\circ} \mathrm{C}$ to the amount of $5 \times 10^{7}$, and were mixed with ice-cold lysis buffer $(1.5 \mathrm{ml})$ and triturated fifty times at $0^{\circ} \mathrm{C}$. The suspension was then centrifuged for $5 \mathrm{~min}$ at $800 \mathrm{~g}$ at $4^{\circ} \mathrm{C}$, and then its supernatant was added to ice-cold medium buffer and centrifuged for $10 \mathrm{~min}$ at $15000 \mathrm{~g}$ at $4^{\circ} \mathrm{C}$. The supernatant thus obtained was used to detect cytochrome $c$ in cytosolic fractions, while the pellet was resuspended in wash buffer and centrifuged for $10 \mathrm{~min}$ at $15000 \mathrm{~g}$ at $4^{\circ} \mathrm{C}$ to remove its supernatant for extracting mtDNA.

\section{Western blot analysis}

Western blot analysis was performed to evaluate cytochrome $c$ protein levels. The cytosolic proteins were subjected to $10 \%$ SDS-PAGE, blotted onto polyvinylidene fluoride membranes (Pall Corporation, Pensacola, FL, USA) and detected by using an enhanced chemiluminescence Western blot kit (Amersham Biosciences, Little Chalfont, Buckinghamshire, UK). Rabbit anti-mouse cytochrome $c$ (sc-7159; Santa Cruz Biotechnology, Santa Cruz, CA, USA) and glyceraldehyde-3-phosphate dehydrogenase (sc-20741; Santa Cruz Biotechnology) polyclonal antibody were purchased from Santa Cruz Biotechnology. The bands were analysed with LabWorks (TM version 4.6; UVP, BioImaging Systems, Upland, CA, USA).

\section{Mitochondrial DNA content assay}

mtDNA was extracted from mitochondria according to the manufacturer's protocol of the General AllGen Kit (Beijing CoWin Biotech Co., Ltd, Beijing, China). Mitochondria were suspended in ice-cold buffer CL $(300 \mu \mathrm{l})$ and agitated for $10 \mathrm{~s}$. RNase A $(1.5 \mu \mathrm{l})$ was added to the suspension and mixed adequately for $45 \mathrm{~min}$ at $37^{\circ} \mathrm{C}$. After standing on ice for $1 \mathrm{~min}$, buffer PP was added to the suspension and agitated for $20 \mathrm{~s}$, and then centrifuged for $3 \mathrm{~min}$ at $12000 \mathrm{~g}$. Its supernatant was suspended to isopropanol and centrifuged for $1 \mathrm{~min}$ at $12000 \mathrm{~g}$. Its pellet was suspended in $70 \%$ alcohol $(300 \mu \mathrm{l})$ and centrifuged for $1 \mathrm{~min}$ at $12000 \mathrm{~g}$. Its supernatant was removed, and mtDNA was dried at room temperature for $15 \mathrm{~min}$. Buffer CL was added to the mtDNA to detect its level with an ultraviolet spectrophotometer.

\section{Statistics}

Statistical analysis was performed using SPSS 12.0 software. Each assay was performed at least three times. Data are expressed as means and standard deviations, and Student's $t$ test was used to determine the significance of differences in multiple comparisons. $P<0.05$ was considered to be statistically significant.

\section{Results}

Nutrient mixture effects on the peripheral blood of aplastic anaemia mice

We used the Sysmex KX-21N Blood Cell analyser to test the effects of nutrient mixture on the peripheral blood of AA mice. After 7 weeks, a significant peripheral blood pancytopenia was evident in AA mice (Table 1). Erythrocytes, leucocytes, $\mathrm{Hb}$ and the platelet mean count were conspicuously reduced to $64 \cdot 5,75 \cdot 3,53 \cdot 4$ and $60 \cdot 2 \%$ of the control mean values, respectively ( $P<0.05$ for all parameters).

The profound effects of the nutrient mixture on AA mice were also observed in the peripheral blood parameters. Erythrocytes, leucocytes, $\mathrm{Hb}$ and platelet counts in the three different doses of the nutrient mixture groups were higher than in the AA group in a dose-dependent manner $(P<0.05 ;$ Table 1$)$. This result showed that the nutrient mixture indeed had an effect on peripheral blood cell levels in AA mice.

\section{Nutrient mixture restores mitochondrial ultrastructure in aplastic anaemia mice}

We performed transmission electron microscopic studies on the bone marrow, spleen and liver of AA mice and found that mitochondrial ultrastructure was clearly affected by the nutrient mixture diet (Fig. 1). The size and shape 
Table 1. The effect of nutrient mixture on the peripheral blood of mice

(Mean values and standard deviations)

\begin{tabular}{|c|c|c|c|c|c|c|c|c|c|}
\hline \multirow[b]{2}{*}{ Group } & \multirow[b]{2}{*}{$n$} & \multicolumn{2}{|c|}{$\begin{array}{l}\text { Erythrocytes } \\
\left(\times 10^{12} / I\right)\end{array}$} & \multicolumn{2}{|c|}{$\begin{array}{l}\text { Leucocytes } \\
\left(\times 10^{9} / \mathrm{l}\right)\end{array}$} & \multicolumn{2}{|c|}{$\mathrm{Hb}(\mathrm{g} / \mathrm{l})$} & \multicolumn{2}{|c|}{ PIt $\left(\times 10^{9} / l\right)$} \\
\hline & & Mean & SD & Mean & SD & Mean & SD & Mean & SD \\
\hline Control & 20 & $10 \cdot 12$ & 1.46 & 9.94 & 1.13 & 159.46 & $15 \cdot 17$ & $871 \cdot 26$ & 273.95 \\
\hline AA & 20 & $3.59^{*}$ & 1.24 & $2.46^{*}$ & 0.52 & $74 \cdot 26^{\star}$ & $13 \cdot 14$ & $346 \cdot 42^{*}$ & 176.58 \\
\hline High dose & 20 & $9.64 \dagger$ & 1.97 & $7.51 \dagger$ & 1.29 & $143.56 \dagger$ & 17.58 & $814 \cdot 71 \dagger$ & 293.56 \\
\hline Middle dose & 20 & $8.07 \dagger$ & 1.56 & 4.13t & 0.47 & $127 \cdot 37 \dagger$ & $16 \cdot 71$ & $754.46 \dagger$ & $210 \cdot 17$ \\
\hline Low dose & 20 & $6.92 \dagger$ & 1.13 & $3.09 \dagger$ & 0.51 & $109.71 \dagger$ & $13 \cdot 26$ & $590 \cdot 13 \dagger$ & $160 \cdot 16$ \\
\hline
\end{tabular}

PIt, platelets; AA, aplastic anaemia.

${ }^{*}$ Mean value was significantly different from that of the control group $(P<0.05)$.

† Mean value was significantly different from that of the AA group $(P<0.05)$.

of mitochondria of the control group were normal. In contrast, in the AA group, mitochondria had enlarged globular structures, accompanied by the disruption or disappearance of the cristae (Fig. 1(a-c)).
Remarkably, mitochondrial ultrastructure was restored in the nutrient mixture groups, compared with the AA group. These results showed that nutrient mixture might promote mitochondrial rehabilitation in AA mice. (a) Bone marrow

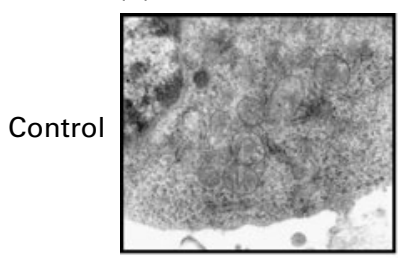

AA

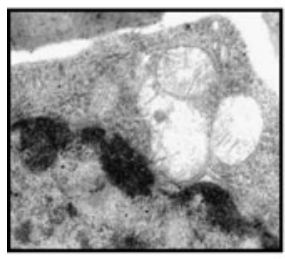

High dose

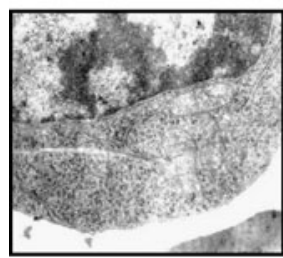

Middle dose
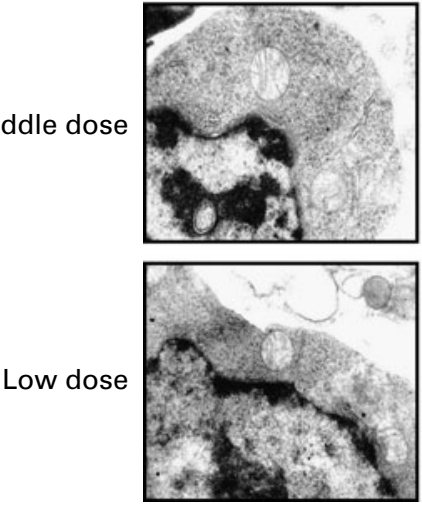

(b) Spleen
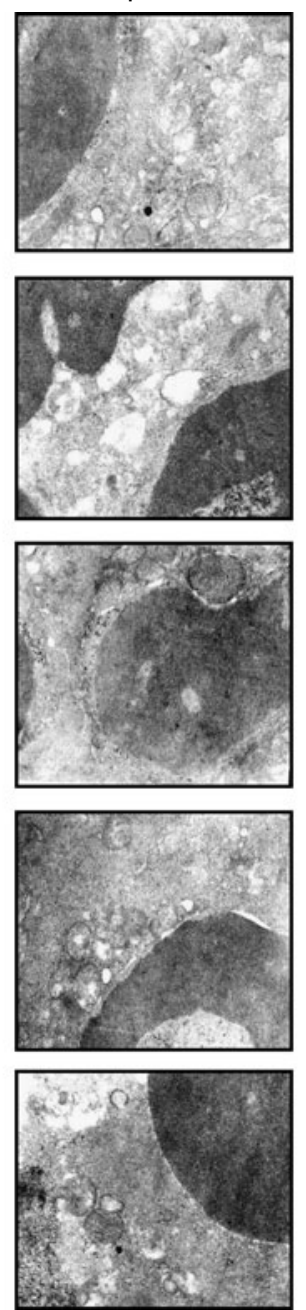

(c) Liver
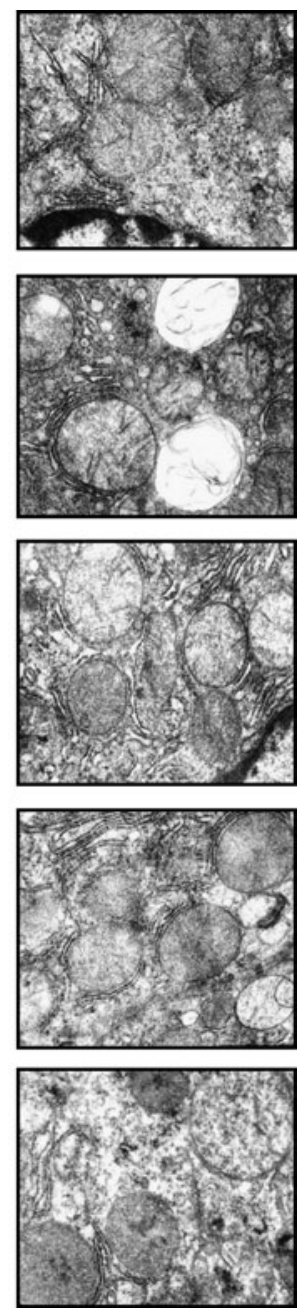

Fig. 1. Effects of nutrient mixture on the mitochondrial morphology of aplastic anaemia (AA) mice. (a) Representative transmission electron micrographs $(20000 \times)$ of mitochondrial structures from haematopoietic cells in the control mice, AA mice and nutrient-mixture-treated AA mice are shown. (b) Representative transmission electron micrographs $(20000 \times)$ of mitochondrial structures from spleens of the control mice, AA mice and nutrient-mixture-treated AA mice are shown. (c) Representative transmission electron micrographs $(20000 \times)$ of mitochondrial structures from livers of the control mice, AA mice and nutrientmixture-treated AA mice are shown. 


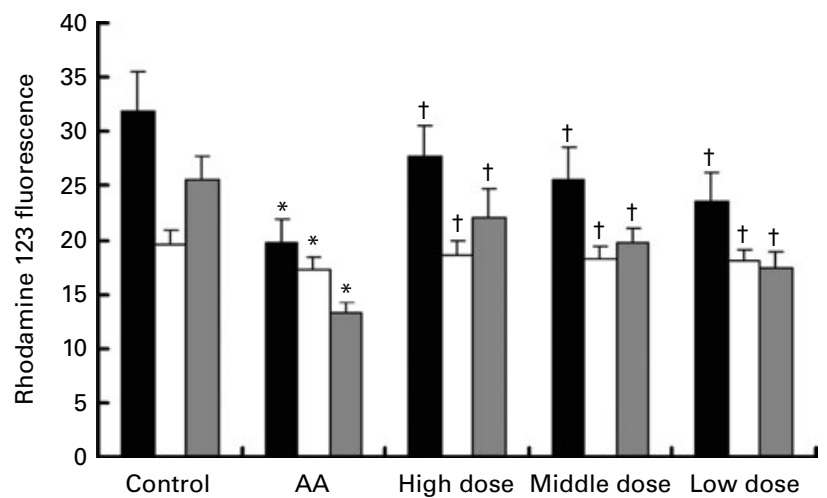

Fig. 2. Effects of nutrient mixture on the mitochondrial membrane potential in cells of aplastic anaemia (AA) mice. $\mathbf{\square}$ : Bone marrow cells from the control mice, AA mice and nutrient-mixture-treated AA mice were stained with rhodamine 123; $\square$ : splenocytes from the control mice, AA mice and nutrientmixture-treated AA mice were stained with rhodamine 123; $\square$ : hepatocytes from the control mice, AA mice and nutrient-mixture-treated AA mice were stained with rhodamine $123\left({ }^{*} P<0.05\right.$ v. control; $† P<0.05$ v. AA).

\section{Nutrient mixture effects on the mitochondrial membrane potential in aplastic anaemia mice}

As transmission electron microscopy findings revealed mitochondrial damage, we assayed through a fluorescence spectrophotometer set to determine the nutrient mixture effect on the MMP in AA mice. We examined the MMP by measuring the relative differences in the fluorescence of Rh123 among three kinds of cells in the five groups. The results showed that the Rh123 fluorescence of bone marrow cells in the AA group (19.7 (SD 2.26)) was lower

(a)

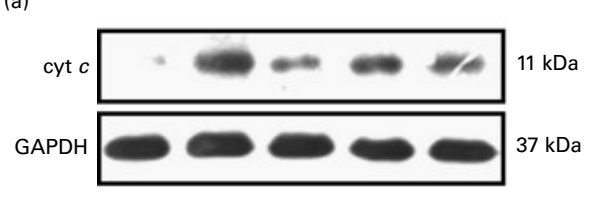

(b)

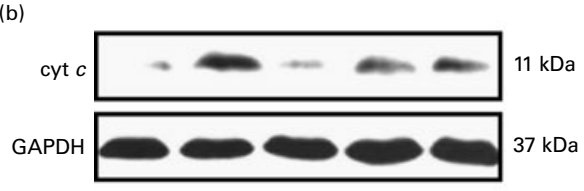

(c)

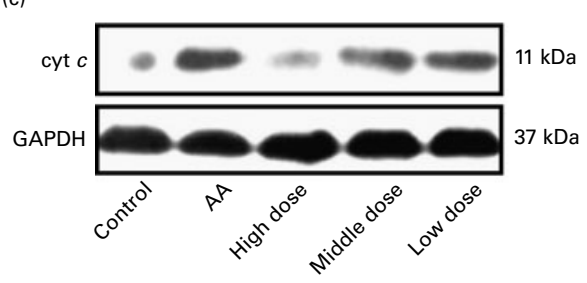

than that in the control group (31.9 (SD 3.62); $P<0.05$ ), and the Rh123 fluorescence of splenocytes in the AA group (17.2 (SD 1.21)) was lower than in the control group (19.6 (sD 1.23); $P<0.05$ ). In addition, the Rh123 fluorescence of hepatocytes in the AA group (25.6 (SD 2.13) was lower than that in the control group (13.2 (SD 1.02); $P<0.05$ ) (Fig. 2). However, the Rh123 fluorescence of the three kinds of cells in the three different doses of the nutrient mixture groups was higher than that in the AA group in a dose-dependent manner $(P<0.05)$ (Fig. 2). This result showed that nutrient mixture affected remarkably the recovery of the MMP of AA mice in a dose-dependent manner.

\section{Nutrient mixture effects on the release of cytochrome c of aplastic anaemia mice}

To examine the effect of nutrient mixture on the release of cytochrome $c$ of AA mice, Western blot analysis was employed to analyse the release of cytochrome $c$, which is from the mitochondria to the cytosol. Cytochrome c expression was variable among the mouse models, with a higher cytochrome $c$ level detected in the cells of the AA group and no detectable level in the cells of the control group. However, the cytochrome $c$ levels of the three kinds of cells in the nutrient mixture groups were markedly lower than that in the AA group (Fig. 3), indicating that nutrient mixture mediated the decrease in cytochrome $c$ levels of AA mice in a dosedependent manner.

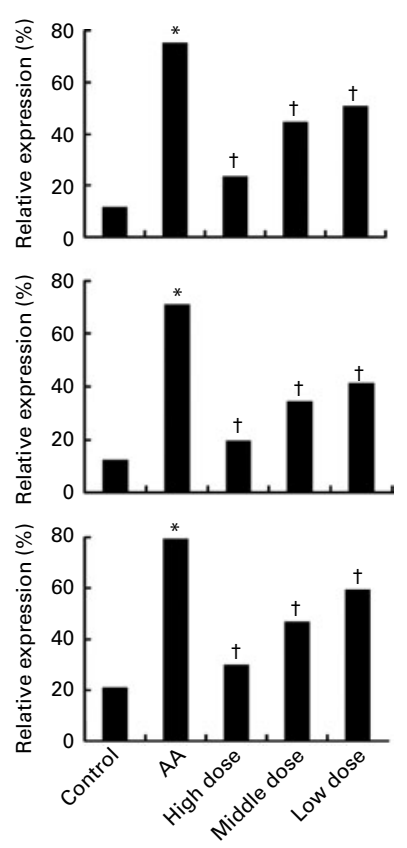

Fig. 3. Effects of nutrient mixture on the cytochrome $c$ (cyt $c$ ) release of mitochondria in aplastic anaemia (AA) mice. The cyt $c$ release assay was performed by Western blot analysis, which was performed on the cytosolic protein fractions of cells (bone marrow cells (a), splenocytes (b) and hepatocytes (c)). After the cells (bone marrow cells, splenocytes and hepatocytes) were lysed, the cytosolic fraction was isolated and SDS-PAGE was carried out. Monoclonal antibody detected an $11 \mathrm{kDa}$ band containing cyt $c$. Glyceraldehyde-3-phosphate dehydrogenase (GAPDH) blotting was used as the control. The relative expression of cytosolic cyt $c$ was expressed as a percentage of GAPDH ( ${ }^{\star} P<0.05$ v. control; $† P<0.05 v$. AA). 


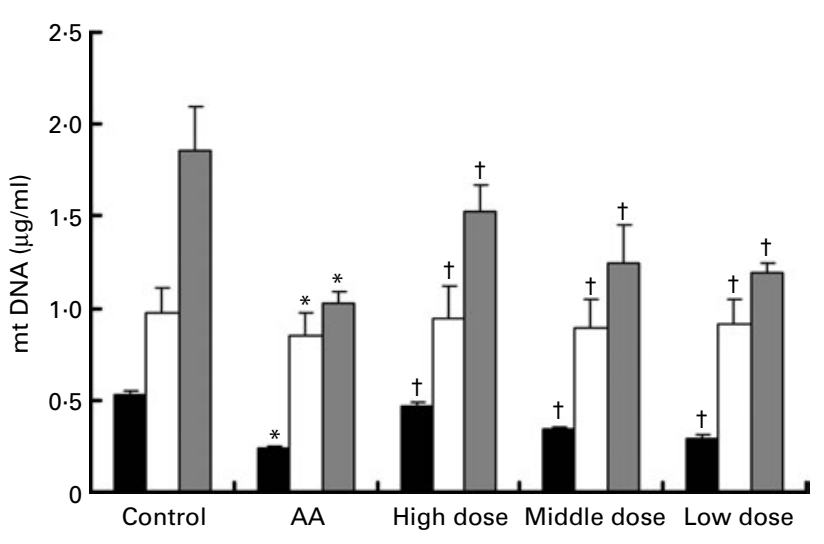

Fig. 4. Effects of nutrient mixture on the mitochondrial DNA content of aplastic anaemia (AA) mice. $\mathbf{\square}$ : Mitochondrial DNA (mtDNA) content of bone marrow cells from the control mice, aplastic anaemia (AA) mice and nutrientmixture-treated AA mice; $\square$ : mtDNA content of splenocytes from the control mice, AA mice and nutrient-mixture-treated AA mice; $\square$ : mtDNA content of hepatocytes from the control mice, $A A$ mice and nutrient-mixture-treated $A A$ mice. ( $P<0.05$ v. control; $† P<0.05$ v. AA).

\section{Nutrient mixture effects on the mitochondrial DNA content of aplastic anaemia mice}

In order to determine whether the nutrient mixture had effects on the mtDNA content of AA mice, an ultraviolet spectrophotometer assay was used to measure mtDNA content in the three kinds of cells in the five groups. The results showed that the mtDNA content of bone marrow cells in the AA group $(0.24(\mathrm{SD} 0.01) \mu \mathrm{g} / \mathrm{ml})$ was lower than that in the control group $(0.53$ (SD 0.02$) \mu \mathrm{g} / \mathrm{ml}$; $P<0.05)$. The mtDNA content of splenocytes in the AA group $(0.85$ (SD 0.12$) \mu \mathrm{g} / \mathrm{ml})$ was lower than that in the control group $(0.98(\mathrm{SD} 0.13) \mu \mathrm{g} / \mathrm{ml} ; P<0.05)$, and the mtDNA content of hepatocytes in the AA group (1.03 (SD $0.06 \mu \mathrm{g} / \mathrm{ml}$ ) was lower than that in the control group (1.86 (sD 0.24$) \mu \mathrm{g} / \mathrm{ml}, P<0.05$ ) (Fig. 4). However, the mtDNA content of the three kinds of cells in the three different doses of the nutrient mixture groups was higher than that in the AA group in a dose-dependent manner $(P<0.05)$ (Fig. 4). These results suggested that the nutrient mixture could promote the recovery of mtDNA content of the AA mice in a dose-dependent manner.

\section{Discussion}

AA is characterised by hypocellular bone marrow resulting from damage to haemopoietic stem cells. This produces pancytopenia with the risk of severe anaemia, major haemorrhage and life-threatening infections. However, the fundamental pathophysiology of AA is not well understood. In the present study, this new animal model of $\mathrm{AA}$ is convenient to use and has the potential for further investigation of roles of the nutrient mixture to mediate the mitochondrial rehabilitation of AA mice.

In our experiments, the AA mouse model was induced by the combination of acetylphenylhydrazine, X-rays and cyclophosphamide. Animals show statistically significant reductions in peripheral blood leucocytes, erythrocytes, $\mathrm{Hb}$ and platelets (Table 1), and even more severe reductions in humeral marrow cells and marrow-committed progenitor cells. These are clinical characteristics of AA. Although many factors are involved in assessing overall health status in patients with AA, nutrition plays a significant role in influencing AA biology, co-morbid conditions and responses to treatment. In the present study, we found that the nutrient mixture could mediate the mitochondrial rehabilitation of AA mice. This stress response, mediated by the complex interaction of nucleotides, arginine, phosphatidylcholine, phosphatidylethanolamine, Mn, Zn, vitamin, folate, GSE powder, etc., can ultimately lead to a nutritional rehabilitation of mitochondrial aberrations in AA mice.

Nucleotides are essential nutrients that modulate cell proliferation and differentiation, lipid metabolism and immune function. Dietary nucleotide supplementation promotes the redox state, efficiency of protein synthesis and restoration of the function and number of mitochondria ${ }^{(13)}$ Amino acids play a significant role in nutrition and disease in humans. Arginine displays crucial metabolic and regulatory versatility in cells. As the physiological nitrogenous precursor for the synthesis of NO, arginine can increase NO synthesis, which mediates the stimulatory action of arginine on the mitochondrial oxidation of energy substrates $^{(14)}$. Glycine prevents hypoxia, free radical formation, cell swelling and the hypermetabolic state ${ }^{(15)}$. Dietary supplementation of patients taking Cylosporin A with glycine should be useful to prevent some side effects of this immunosuppressive drug ${ }^{(15,16)}$. Humans and other mammals cannot synthesise vitamins and folate, so they must be obtained from exogenous sources via absorption in the intestine. Vitamins are important micronutrients for humans. Vitamin E is the major lipid-soluble, chain-breaking antioxidant and plays an essential role in maintaining the integrity of biological membranes, reproduction, preventing lipid peroxidation and other radical-driven oxidative events ${ }^{(17)}$. As a factor, vitamin $\mathrm{E}$ participates in the mitochondrial electron transport system, and vitamin $\mathrm{E}$ deficiency may cause a respiratory decline in mitochondria $^{(18)}$. So dietary vitamin E can prevent mitochondrial damage and restore mitochondrial function. As an aqueous free radical trap, vitamin $\mathrm{C}$ can increase the antioxidant effectiveness of vitamin $\mathrm{E}$; however, it is itself a kind of antioxidant ${ }^{(19)}$. The effect of vitamin $C$ on viability, reactive oxygen species and MMP has been determined ${ }^{(19)}$. Vitamin $\mathrm{B}_{6}$ deficiency affects ultrastructural abnormalities in the central nervous system. On account of vitamin $\mathrm{B}_{12}$ containing the metal cobalt, it is also called cobalamin. An adequate supply of vitamin $\mathrm{B}_{12}$ and folic acid is essential for human health and well-being; thus there is a variety of clinical abnormalities that can occur as a result of vitamin $\mathrm{B}_{12}$ and folic acid deficiency ${ }^{(20,21)}$. $\mathrm{Zn}$ is an essential cofactor that serves a variety of biochemical processes including cellular respiration, cellular utilisation of oxygen, DNA and 
RNA, reproduction, maintenance of cell membrane integrity and sequestration of free radicals ${ }^{(22)}$. As an enzyme activator, Mn plays a significant role in enzymatic synthesis, and $\mathrm{Mn}$ deficiency can result in membrane damage, which is due to the accumulation of free $\mathrm{O}_{2}^{-}$inside the mitochondria $^{(23)}$. Fe, a kind of microelement, is related to biological function. Fe deficiency can result in mitochondrial dysfunction and mtDNA damage ${ }^{(24)}$. GSE is a complex mixture of polyphenols including procyanidins and proanthocyanidins. As a free radical scavenger, GSE has a protective effect on oxidant-induced production and deposition of extracellular matrix components ${ }^{(25)}$.

In the present study, AA mice were treated with the nutrient-mixture-supplemented diets in a dose-dependent manner including the above-mentioned nutritive substances, and changes were measured. The results presented in Table 1 demonstrated that AA mice treated with the nutrient-mixture-supplemented diets appear to be sensitive to the nutrient mixture, and the haematological parameters of these animals become more significantly affected than the changes observed in the AA group. There was a very significant increase in $\mathrm{Hb}$, erythrocytes, leucocytes and platelet counts $(P<0.05 v$. AA group). This result showed that the nutrient mixture had indeed an effect on peripheral blood cell levels in AA mice.

Mitochondria are double-membrane organelles that play a major role in providing energy and in regulating osmotic pressure, $\mathrm{Ca}$ homeostasis and cell signalling ${ }^{(8)}$. Mitochondrial aberration is often accompanied by changes in mitochondrial morphology. The results demonstrated that the mitochondria of bone marrow cells, splenocytes and hepatocytes were swollen, and the cristae were fragmented and solubilised in the AA group. However, the structure of mitochondria in different doses of the nutrient mixture groups was restored (Fig. 1). This is consistent with previously reported results that dietary nutrient mixtures can promote mitochondrial rehabilitation ${ }^{(17-19)}$.

MMP, a significant indicator of cellular viability, is important for an integrated appraisal of mitochondrial function $^{(15)}$. Cytochrome $\mathrm{c}$ is a critical factor in the apoptotic process, and rupture of the mitochondrial membrane can result in the release of cytochrome $c$ from the mitochondria into the cytosol ${ }^{(26)}$. We first examined cytochrome $c$ release using Western blot analysis. We found that the nutrient mixture inhibited cytochrome $c$ release (Fig. 3), and that the nutrient mixture changed the MMP (Fig. 2). We also found that dietary supplementation of AA mice with the nutrient mixture mediated these changes in a dose-dependent manner. Similar results were demonstrated that vitamin $\mathrm{B}_{6}$ supplementation prevented the altered MMP in U937 monocytes ${ }^{(27)}$.

Mutations and the instability of mtDNA have recently been demonstrated in a wide variety of diseases. Mitochondrial dysfunction and the decrease in the amount of mitochondria may result in the reduction of mtDNA. The present results showed that the nutrient mixture could restore the mtDNA content in a dose-dependent manner, in accordance with the previously reported results that folic acid supplementation may be protective against mtDNA deletions ${ }^{(28)}$.

On the basis of the above-mentioned analysis, the present study has demonstrated that the nutrient mixture promotes the rehabilitation of mitochondrial aberrations in AA mice. To the best of our knowledge, this is the first study to evaluate a nutrient mixture for its nutritional rehabilitation of mitochondrial aberrations, and consequently protects against mitochondrial dysfunction.

\section{Acknowledgements}

We thank W. W. for providing the nutrient mixture. Financial support was provided by the Dalian Municipal Science and Technology Plan Project (special item of innovative drugs). L. H. and L. J. had the idea for the study. L. H., P. Y. and L. J. conceived, conceptualised and supervised the study. L. H. performed the experiments. L. H., X. M. and G. L. performed the analysis and interpreted the data. X. M. and G. L. contributed reagents/materials/analysis tools. L. J. revised the manuscript critically. None of the authors had any conflicts of interest.

\section{References}

1. Young NS (2002) Acquired aplastic anemia. Ann Intern Med 136, 534-546.

2. Laveder F \& Marcolongo R (1996) Uncontrolled triggering of programmed cell death (apoptosis) in haematopoietic stem cells: a new hypothesis for the pathogenesis of aplastic anaemia. Immunol Cell Biol 74, 159-162.

3. Benzi G, Corona GL \& Frico GM (1964) Effect of cobalt on anemia caused by acetylphenylhydrazine. I. Research in normal rats. Boll Soc Ital Biol Sper 40, 1289-1292.

4. Knospe WH, Blom J \& Crosby WH (1968) Regeneration of locally irradiated bone marrow. II. Induction of regeneration in permanently aplastic medullary cavities. Blood 31, $400-405$.

5. Stojanović N, Milenković P, Jovcić G, et al. (1989) Polycythemic mice - an appropriate model for investigation of granulopoiesis. Haematologica 74, 19-24.

6. Naviaux RK (2000) Mitochondrial DNA disorders. Eur J Pediatr 159, S219-S226.

7. Seppet E, Gruno M, Peetsalu A, et al. (2009) Mitochondria and energetic depression in cell pathophysiology. Int J Mol Sci 10, 2252-2303.

8. He L, Luo L, Proctor SJ, et al. (2003) Somatic mitochondrial DNA mutations in adult-onset leukaemia. Leukemia 17, 2487-2491.

9. Gattermann N (2004) Mitochondrial DNA mutations in the hematopoietic system. Leukemia 18, 18-22.

10. Gattermann N, Retzlaff S, Wang YL, et al. (1997) Heteroplasmic point mutations of mitochondrial DNA affecting subunit I of cytochrome $c$ oxidase in two patients with acquired idiopathic sideroblastic anemia. Blood 90, 4961-4972.

11. Kim HR, Shin MG, Kim MJ, et al. (2008) Mitochondrial DNA aberrations of bone marrow cells from patients with aplastic anemia. Korean Med Sci 23, 1062-1067. 
12. Johnson LV, Walsh ML \& Chen LB (1980) Localization of mitochondria in living cells with rhodamine 123. Proc Natl Acad Sci U S A 77, 990-994.

13. Pırez MJ, S $\alpha$ nchez-Medina F, Torres M, et al. (2004) Dietary nucleotides enhance the liver redox state and protein synthesis in cirrhotic rats. J Nutr 134, 2504-2508.

14. Fu WJ, Haynes TE, Kohli R, et al. (2005) Dietary L-arginine supplementation reduces fat mass in Zucker diabetic fatty rats. J Nutr 135, 714-721.

15. Zhong Z, Li X, Yamashina S, et al. (2001) Cyclosporin A causes a hypermetabolic state and hypoxia in the liver: prevention by dietary glycine. J Pharmacol Exp Ther 299, $858-865$.

16. Zhong Z, Arteel GE, Connor HD, et al. (1998) Cyclosporin A increases hypoxia and free radical production in rat kidneys: prevention by dietary glycine. Am J Physiol 275, F595-F604.

17. Brigelius-Flohı R \& Traber MG (1999) Vitamin E: function and metabolism. FASEB J 13, 1145-1155.

18. Chen LH \& Chang ML (1978) Effect of dietary vitamin E and vitamin $\mathrm{C}$ on respiration and swelling of guinea pig liver mitochondria. J Nutr 108, 1616-1620.

19. Heaney ML, Gardner JR, Karasavvas N, et al. (2008) Vitamin $\mathrm{C}$ antagonizes the cytotoxic effects of antineoplastic drugs. Cancer Res 68, 8031-8038.

20. Hanna S, Lachover L \& Rajarethinam RP (2009) Vitamin b(12) deficiency and depression in the elderly: review and case report. Prim Care Companion J Clin Psychiatry 11, 269-270.
21. Said HM, Chatterjee N, Haq RU, et al. (2000) Adaptive regulation of intestinal folate uptake: effect of dietary folate deficiency. Am J Physiol Cell Physiol 279, C1889-C1895.

22. Yousef MI, El-Hendy HA, El-Demerdash FM, et al. (2002) Dietary zinc deficiency induced-changes in the activity of enzymes and the levels of free radicals, lipids and protein electrophoretic behavior in growing rats. Toxicology 175, 223-234.

23. de Rosa G, Keen CL, Leach RM, et al. (1980) Regulation of superoxide dismutase activity by dietary manganese. $J$ Nutr 110, 795-804.

24. Walter PB, Knutson MD, Paler-Martinez A, et al. (2002) Iron deficiency and iron excess damage mitochondria and mitochondrial DNA in rats. Proc Natl Acad Sci U S A 99, 2264-2269.

25. Dulundu E, Ozel Y, Topaloglu U, et al. (2007) Grape seed extract reduces oxidative stress and fibrosis in experimental biliary obstruction. J Gastroenterol Hepatol 22, 885-892.

26. Kluck RM, Bossy-Wetzel E, Green DR, et al. (1997) The release of cytochrome $c$ from mitochondria: a primary site for Bcl-2 regulation of apoptosis. Science 275, 1132-1136.

27. Kannan K \& Jain SK (2004) Effect of vitamin $B_{6}$ on oxygen radicals, mitochondrial membrane potential, and lipid peroxidation in $\mathrm{H}_{2} \mathrm{O}_{2}$-treated U937 monocytes. Free Radic Biol Med 36, 423-428.

28. Branda RF, Brooks EM, Chen Z, et al. (2002) Dietary modulation of mitochondrial DNA deletions and copy number after chemotherapy in rats. Mutat Res 501, 29-36. 